SUPPORTING INFORMATION

\title{
An immunotherapy strategy targeting programmed cell death ligand 1 and CD73 with macrophage-derived mimetic nanovesicles to treat bladder cancer
}

Qidong Zhou ${ }^{a, b \neq t}$, Weihong Ding ${ }^{a, b \neq}$, Zhiyu Qian ${ }^{a, b \neq}$, Quangang Zhu ${ }^{c}$, Chuanyu Sun ${ }^{a, b}$, Qin $Y u^{c}$, Zongguang Tai ${ }^{c *}, K e X u^{a, b *}$

aDepartment of Urology, Huashan Hospital, Fudan University, Shanghai 200040,

China

bFudan Institute of Urology, Huashan Hospital, Fudan University, Shanghai 200040, China

'Shanghai Skin Disease Hospital, School of Medicine, Tongji University, Shanghai 200443, China 
Table S1. The aPDL1 contents of the EMVs-aPDL1 at various EMVs/aPDL1 ratios

Weight ratio of EMVs to aPDL1

Graft ratio of aPDL1 content

(mg protein/mg, w/w)

$(\%, \mathrm{w} / \mathrm{w})$

1.0

$2.49 \pm 0.18$

1.5

$3.81 \pm 0.26$

2.0

$4.72 \pm 0.30$

$2.5^{*}$

$5.47 \pm 0.36$

3.0

$5.50 \pm 0.24$

3.5

$5.45 \pm 0.19$

*The 2.5 weight ratio of EMVs/aPDL1 (mg protein/mg, w/w) was the best option for constructing the EMVs-aPDL1 nanoparticles.

EMVs: exosome-mimetic nanovesicles, aPDL1: monoclonal antibody targeting programmed cell death ligand 1 . 